\title{
TOPOLOGICAL QUANTIZATION OF FREE MASSIVE BOSONIC FIELDS
}

\author{
GUSTAVO ARCINIEGA, FRANCISCO NETTEL, LEONARDO PATINO \\ AND HERNANDO QUEVEDO
}

Communicated by Jean-Francois Ganghoffer

\begin{abstract}
We present the results of studying free massive bosonic fields under the formalism of topological quantization. We identify certain harmonic maps as a geometric configuration equivalent to the classical system. We take as a concrete example the case of free massive bosonic fields in two dimensions, and construct the fiber bundle representing them and find its topological spectra. We found that the appearance of singularities in Euler's two form caused its integral to be dependent on the order in which the variables are integrated. We discuss the implications of this orientation dependency and formulate a well-defined expression for the Euler invariant emerging from it.
\end{abstract}

\section{Introduction}

The formalism of topological quantization in the way we will understand it here was formulated in $[7,12]$. In general, this formalism, has been constructed to find the discrete behaviour of physical quantities through topological properties associated to the physical system.

In order to apply this formalism we need a geometrical configuration which must be equivalent to the physical system we want to analyze, this means that the geometrical configuration must encode all physical information of the physical system. According to Patiño and Quevedo, we can apply this formalism in two ways: a) intrinsic topological quantization and $b$ ) induced topological quantization.

Both ways analyze the properties of an associated principal fiber bundle (PFB). The topological quantization is called intrinsic when the structure group of the PFB to be studied is determined by the intrinsic symmetries of the base space. In this case, the resulting PFB is equivalent to that of the tangent bundle. In the intrinsic topological quantization, we need a base space manifold $M$ covered by open sets $U_{i}$ and a metric $g$. The connection $\tilde{\omega}$ on the tangent bundle $T M$, coming from the lifting of the metric connection $\omega$ on $M$ is the one satisfying the relation $\sigma_{i}^{*} \tilde{\omega}=\omega_{i}$ for any section $\sigma_{i}$ defined over $U_{i}$, and the asterisk denotes the pullback 
of a mapping. To analyze the topology of $T M$ we can use $\tilde{\omega}$ to compute the characteristic class of the corresponding PFB $C(P)=C(T M)$. The details of how to perform this calculation will depend on the signature and dimensionality of $M$, but the relevant part here is to notice that the integral of $C(T M)$ over $M$ is bound to give an integer, i.e.,

$$
\int C(T M)=\chi, \quad \chi \in \mathbb{Z} .
$$

The induced topological quantization takes its name from the fact that the fiber bundle to be analyzed and its topological characteristics are dictated by a physical gauge field living on the base space, so its standard fiber is not directly related to the the tangent space at each point. A prominent example of this type of quantization is that of Dirac's monopole. In this case, the gauge field is commonly a one-form $\mathbf{A}^{1}$ with components taking values in the Lie algebra $\mathfrak{g}$ of the structure group $G$. The discrete conditions arise when we impose the regularity condition on the gauge field to be well-defined over the entire PFB.

Let us remember that all the information of the physical system is contained in the geometric construction, so, at least a fraction of it will be captured in $M$ and its metric $g$, information that we could capture in some parameters that we can call $a_{\alpha}$. This part of the information will be carried to $C(T M)$, so this will depend on the parameters $a_{\alpha}$ and the point $p$ of the manifold where it is evaluated. Being $\chi$ the integral of $C(T M)$ over the manifold, it will only depend on the parameter $a_{\alpha}$ so it can be written as a function of the parameters, namely $f\left(a_{\alpha}\right)$. Using (1) we can write

$$
f\left(a_{\alpha}\right)=\chi, \quad \chi \in \mathbb{Z} .
$$

This equation is what we called the topological spectrum and what gives us the chance to analyze the physical meaning it could carry.

In this work, we will illustrate how to apply this formalism of topological quantization in a particular case of free massive bosonic fields. Once we choose the physical system, we can split the entire formalism in three steps:

1) Construct a geometrical configuration equivalent to that physical system which is also called classical configuration in [7,8] (Section 3)

2) Construct the PFB and compute the topological spectrum (Section 4 and Section 5)

\footnotetext{
${ }^{1}$ This can be easily generalized to higher dimensions when the gauge field $\mathbf{A}$ is a $n$-form as is shown in $[10,17]$.
} 
3) Analyze the physical meaning of the topological spectrum (Section 6).

\section{Free Massive Bosonic Fields}

The first step to apply this formalism is to find a geometric configuration that represents the physical system under consideration from which we will construct a PFB over it. This formulation of topological quantization was presented and applied for the first time to gravitational configurations in $[11,12]$, after that it was carried out in [7-9] to classical systems of $n$-degrees of freedom and recently was applied to bosonic strings in different backgrounds in [1]. In this work, we are interested in the free massive bosonic fields $X^{i}, i=1, \ldots, d$, where $i$ is the number of fields considered, which has the associated action $S$ given by [14]

$$
S=\frac{1}{2} \int \sum_{i}^{d}\left(\partial^{\mu} X^{i} \partial_{\mu} X^{i}-m^{2} X^{i}\right) \mathrm{d} x^{n}
$$

where $\mu=1, \ldots, n$ and $n$ is the dimension of the spacetime and $m$ is the mass term associated to each field.

The variation of this action leads us to the equation of motion

$$
\left(\partial^{\mu} \partial_{\mu}+m^{2}\right) X^{i}=0 .
$$

In equation (3) we have encoded all the dynamical information of the physical system.

\section{Harmonic Maps}

Consider two differential manifolds $M$ and $N$ with metrics $g(x)$ and $G(X)$, respectively, where $x$ and $X$ are the corresponding local coordinates. A harmonic map is a smooth mapping $\mathbf{X}: M \mapsto N$ such that it satisfies the equations of motion that follow from the variation of the Dirichlet energy functional

$$
S_{h}=\int \sqrt{g} g^{a b}(x) \partial_{a} X^{i} \partial_{b} X^{j} G_{i j}(X) \mathrm{d}^{m} x .
$$

This set of equations is

$$
\frac{1}{\sqrt{g}} \partial_{a}\left(g^{a b} \partial_{b} X^{i}\right)+\Gamma_{j k}^{i}(X) \partial_{a} X^{j} \partial_{a} X^{k} g^{a b}=0
$$


where $\Gamma_{j k}^{i}$ are the Christoffel symbols for $G_{i j}$. We can understand the mapping $\mathbf{X}$ as a minimal embedding of $M$ into $N$. Now, if we take $M$ to be a two-dimensional differential manifold with metric $g_{a b}$ given by

$$
g_{a b}=\eta_{a b}
$$

where $\eta_{a b}$ is the Minkowskian two-dimensional metric $(\eta=\operatorname{diag}(-1,1))$ and we denote the coordinates as $\tau$ and $\sigma$. The target space $N$ is a ten-dimensional differential manifold with a metric $G$ which components in terms of the lightcone coordinates $X^{+}=\frac{1}{\sqrt{2}}\left(X^{0}+X^{1}\right), X^{-}=\frac{1}{\sqrt{2}}\left(X^{0}-X^{1}\right)$ and $X^{I=1, \ldots, 8}$ are respectively $[4,15,16]$

$$
\begin{aligned}
G_{+-} & =G_{-+}=-1, \quad G_{--}=0, \quad G_{++}=-\mu^{2} \sum_{I=1}^{8} X^{I} X^{I} \\
G_{I J} & =\delta_{I J}, \quad I, J=1, \ldots, 8 .
\end{aligned}
$$

In the light cone coordinates, the explicit form of the action (4) is

$$
\begin{aligned}
S= & -\frac{1}{4 \pi \alpha^{\prime}} \int \sqrt{-g} g^{a b}\left[-2 \partial_{a} X^{+} \partial_{b} X^{-}+\sum_{I=1}^{8} \partial_{a} X^{I} \partial_{b} X^{I}\right. \\
& \left.-\mu^{2}\left(\sum_{I=1}^{8} X_{I} X^{I}\right) \partial_{a} X^{+} \partial_{b} X^{+}\right] \mathrm{d} \sigma \mathrm{d} \tau .
\end{aligned}
$$

We will use the light-cone gauge given by

$$
X^{+}=\alpha^{\prime} p^{+} \tau, \quad p^{+} \geq 0 .
$$

Using (5) and (8) we fix all gauge symmetries of the action and vary the action with respect to the metric $g_{a b}$, i.e.,

$$
\frac{\delta S}{\delta g^{\tau \sigma}}=0, \quad \frac{\delta S}{\delta g^{\tau \tau}}=-\frac{\delta S}{\delta g^{\sigma \sigma}}=0 .
$$

In this way we obtain

$$
\begin{aligned}
& \partial_{\sigma} X^{-}=\frac{1}{\alpha p^{+}} \sum_{I=1}^{8} \partial_{\sigma} X^{I} \partial_{\tau} X^{I} \\
& \partial_{\tau} X^{-}=\frac{1}{2 \alpha p^{+}} \sum_{I=1}^{8}\left[\partial_{\tau} X^{I} \partial_{\tau} X^{I}+\partial_{\sigma} X^{I} \partial_{\sigma} X^{I}-\left(\mu \alpha^{\prime} p^{+}\right)^{2} X^{I} X^{I}\right]
\end{aligned}
$$


From the above equations, we can see that $X^{-}$is not a dynamical variable, and considering (8), we can write the action (7) as

$$
S=-\frac{1}{4 \pi \alpha^{\prime}} \iint_{0}^{2 \pi \alpha^{\prime} p^{+}} \sum_{I=1}^{8}\left[-\partial_{\tau} X^{I} \partial_{\tau} X^{I}+\partial_{\sigma} X^{I} \partial_{\sigma} X^{I}+\mu^{2} X_{I} X^{I}\right] \mathrm{d} \sigma \mathrm{d} \tau
$$

where we rescaled $\tau$ and $\sigma$ by $\alpha^{\prime} p^{+}$and periodic conditions on $\sigma$ have been imposed. Observe that we can describe eight non-interacting massive Klein-Gordon fields in two dimensions as the harmonic map given by (10).

We notice that there are two manifolds involved in the equivalent geometric system, so both are in principle subject to the procedure of topological quantization.

\section{Topological Spectrum From the Target Space}

The metric (6) together with the five-form

$$
F_{+1234}=F_{+5678}=2 \mu
$$

and a constant dilation field

$$
\phi=\text { const. }
$$

constitute a solution of Supergravity IIB [15] representing a pp-wave [13].

For this space-time, a quick calculation shows that Euler's form computed from the metric connection associated to (6) turns out to be zero, and therefore the intrinsic topological quantization does not impose any restrictions.

Interestingly, the five-form (11) is known to be subject to Dirac quantization [3,6, $10,17]$ this leads to the condition

$$
\mu=\phi \sqrt{(\pi / 2) n}, \quad n \in \mathbb{Z}
$$

which will be used later in this work.

\section{Topological Quantization From the Embedded Space}

The other possibility to construct a PFB is to take the embedded manifold as a base space with induced metric given by

$$
h_{a b}=\partial_{a} X^{i} \partial_{b} X^{j} G_{i j}
$$

Using (8) and (9), the induced metric is conformally flat

$$
h_{a b}=f \eta_{a b}=f\left(\begin{array}{cc}
-1 & 0 \\
0 & 1
\end{array}\right)
$$


where $f=\sum_{I=1}^{8} \partial_{\sigma} X^{I} \partial_{\sigma} X^{I}$.

We can now carry the intrinsic topological quantization of the embedded manifold with metric (12). We will use for the tangent space at each point a semiorthonormal basis $\mathbf{e}^{\mu}=e_{a}^{\mu} \mathrm{d} \sigma^{a}, \sigma^{a}=\{\tau, \sigma\}$, satisfying

$$
e_{a}^{\mu} e_{b}^{\nu} \eta_{\mu \nu}=h_{a b}
$$

and accordingly, the spin connection $\omega$ in the standard way

$$
\left(\omega^{\mu}{ }_{\nu}\right)_{a}=e_{b}^{\mu} \partial_{a} e_{\nu}^{b}+e_{b}^{\mu} \Gamma_{a c}^{b} e_{\nu}^{c}
$$

Given the signature and dimensionality of $M$, the components $\left(\omega^{\mu \nu}\right)$ of the oneform $\omega$ take values in the Lie algebra of $\operatorname{SO}(1,1)$, therefore the characteristic class we need to compute is the Euler class $e(T M)$ given by

$$
e(T M)=\frac{-1}{4 \pi} \epsilon_{\mu}^{\nu} \mathbf{R}_{\nu}^{\mu}
$$

where $\epsilon_{\mu}^{\nu}$ is the Levi-Civita tensor and $\mathbf{R}_{\nu}^{\mu}$ is the curvature two-form $R_{\nu a b}^{\mu} \mathrm{d} \sigma^{a} \wedge \mathrm{d} \sigma^{b}$ with components

$$
R_{\nu a b}^{\mu}=\partial_{a} \omega_{\nu b}^{\mu}-\partial_{b} \omega_{\nu a}^{\mu}+\omega_{\gamma a}^{\mu} \omega_{\nu b}^{\gamma}-\omega_{\gamma b}^{\mu} \omega_{\nu a}^{\gamma} .
$$

From the metric (13), the expression (14) simplifies to

$$
e(T M)=\frac{1}{4 \pi}\left[\partial_{\sigma}\left(\frac{\partial_{\sigma} f}{f}\right)-\partial_{\tau}\left(\frac{\partial_{\tau} f}{f}\right)\right] \mathrm{d} \tau \wedge \mathrm{d} \sigma .
$$

Thus, to compute $f$, we make use of the explicit expression for the fields $X^{I}$. The equation of motion for each $X^{I}$ is

$$
\left(\partial_{\tau}^{2}-\partial_{\sigma}^{2}+\mu^{2}\right) X^{I}=0
$$

with general solution, satisfying the right periodic conditions, given by

$$
\begin{aligned}
X^{I}= & x_{0}^{I} \cos \mu \tau+\frac{p_{0}^{I}}{\mu p^{+}} \sin \mu \tau \\
& +\sqrt{\frac{\alpha^{\prime}}{2}} \sum_{n=1}^{\infty} \frac{1}{\sqrt{\omega_{n}}}\left[\alpha_{n}^{I} \mathrm{e}^{-\mathrm{i} \tilde{\omega}_{n}^{+}}+\tilde{\alpha}_{n}^{I} \mathrm{e}^{-\mathrm{i} \tilde{\omega}_{n}^{-}}+\alpha_{n}^{\dagger I} \mathrm{e}^{\mathrm{i} \tilde{\omega}_{n}^{+}}+\tilde{\alpha}_{n}^{\dagger I} \mathrm{e}^{\mathrm{i} \tilde{\omega}_{n}^{-}}\right]
\end{aligned}
$$

where

$$
\tilde{\omega}_{n}^{+}=\frac{\omega_{n}+n \sigma}{\alpha^{\prime} p^{+}}, \quad \tilde{\omega}_{n}^{-}=\frac{\omega_{n}-n \sigma}{\alpha^{\prime} p^{+}}, \quad \omega_{n}=\sqrt{n^{2}+\left(\mu \alpha^{\prime} p^{+}\right)^{2}}, \quad n \in \mathbb{N}
$$


and $\alpha_{n}^{I}, \tilde{\alpha}_{n}^{I}$ are the coefficients of the right and left modes, respectively.

From the above solutions, we observe that the values we assign to the $\alpha_{n}^{I}$ and $\tilde{\alpha}_{n}^{I}$ will determine $e(T M)$ and so, its integral over $M$

$$
\int_{M} e(T M)=\xi_{T M} .
$$

Then the characteristic invariant will be a function $\xi_{T M}\left(\alpha_{n}, \tilde{\alpha}_{n}\right)$ of these coefficients.

From the Gauss-Bonnet theorem applied to (17), we know that $\xi_{T M}\left(\alpha_{n}, \tilde{\alpha}_{n}\right) \in \mathbb{Z}$, and this condition is the one determining the topological spectrum for the physical parameters $\alpha_{n}, \tilde{\alpha_{n}}$ and $\omega_{n}$.

What is left for us to do now is to compute the explicit form of $\xi_{T M}\left(\alpha_{n}, \tilde{\alpha}_{n}\right) \in \mathbb{Z}$, extract the topological spectrum and analyze its physical content.

It would seem natural to try to integrate (15) over $M$ in its more general form to get the topological spectrum. Nonetheless, by doing so we would get one single condition $\xi_{T M}\left(\alpha_{n}, \tilde{\alpha_{n}}\right) \in \mathbb{Z}$ for an infinite set of $\alpha$ 's and $\tilde{\alpha}$ 's coefficients.

Alternatively, we can start by considering the solutions (16) with the fewest number of coefficients different from zero that still give a non-trivial result for $\xi_{T M}\left(\alpha_{n}, \tilde{\alpha_{n}}\right)$ and extract a condition from each of these cases. The topological spectrum will be the set of values that satisfy all the constrains obtained by iterating this process.

The simplest solution leading to a non-trivial Euler invariant is given by (16) with only $\alpha_{n}^{1}$ and $\tilde{\alpha}_{-n}^{2}$ different from zero, hence all the fields $X^{i \neq\{1,2\}}$ vanish identically, while for $X^{1}$ and $X^{2}$ we have

$$
\begin{aligned}
& X^{1}=x_{0}^{1} \cos (\mu \tau)+\frac{p_{0}^{1}}{\mu p^{+}} \sin (\mu \tau)+\sqrt{\frac{\alpha^{\prime}}{2}} \frac{2 r_{n}}{\sqrt{\omega_{n}}} \cos \left[\tilde{\omega}_{n}^{+}+\gamma\right] \\
& X^{2}=x_{0}^{2} \cos (\mu \tau)+\frac{p_{0}^{2}}{\mu p^{+}} \sin (\mu \tau)+\sqrt{\frac{\alpha^{\prime}}{2}} \frac{2 \tilde{r}_{n}}{\sqrt{\omega_{n}}} \cos \left[\tilde{\omega}_{n}^{-}+\tilde{\gamma}\right]
\end{aligned}
$$

where we wrote $\alpha_{n}^{1}$ and $\tilde{\alpha}_{-n}^{2}$ in their polar representation $r_{n} \mathrm{e}^{-\mathrm{i} \gamma}$ and respectively $\tilde{r}_{n} \mathrm{e}^{-\mathrm{i} \tilde{\gamma}}$.

The Euler form of this field configuration is

$$
\begin{aligned}
e(T M)= & \left\{r _ { n } ^ { 2 } \tilde { r } _ { n } ^ { 2 } \left[\left(\omega_{n}^{2}-n^{2}\right)\left(\cos \left[2\left(\tilde{\omega}_{n}^{+}+\gamma\right)\right]\right)+\cos \left[2\left(\tilde{\omega}_{n}^{-}+\tilde{\gamma}\right)\right]\right.\right. \\
& \left.-2 \omega_{n}^{2} \cos \left(2(\gamma-\tilde{\gamma})+\frac{4 n \sigma}{\alpha^{\prime} p^{+}}\right)+2 n^{2} \cos \left(2(\gamma+\tilde{\gamma})+\frac{4 \omega_{n} \tau}{\alpha^{\prime} p^{+}}\right)\right] \\
& \left.-2\left(\omega_{n}^{2}-n^{2}\right)\left[r_{n}^{4} \sin ^{2}\left(\tilde{\omega}_{n}^{+}+\gamma\right)+\tilde{r}_{n}^{4} \sin ^{2}\left(\tilde{\omega}_{n}^{-}+\tilde{\gamma}\right)\right]\right\} / \\
& \left\{2 \pi\left(\alpha^{\prime} p^{+}\right)^{2}\left[r_{n}^{2} \sin ^{2}\left(\tilde{\omega}_{n}^{+}+\gamma\right)+\tilde{r}_{n}^{2} \sin ^{2}\left(\tilde{\omega}_{n}^{-}+\tilde{\gamma}\right)\right]^{2}\right\} \mathrm{d} \tau \wedge \mathrm{d} \sigma .
\end{aligned}
$$


In order to integrate this expression over $M$, we make the change of variables

$$
x=\sin \left(\tilde{\omega}_{n}^{+}+\gamma\right), \quad y=\sin \left(\tilde{\omega}_{n}^{-}+\tilde{\gamma}\right)
$$

in terms of which the Euler form acquires the form

$$
\begin{aligned}
e(T M)= & \left\{\left(n^{2}-\omega_{n}^{2}\right)\left(r_{n}^{4} x^{2}+\tilde{r}_{n}^{4} y^{2}\right)-n^{2} r_{n}^{2}\left[y^{2}+4 x y \sqrt{1-x^{2}} \sqrt{1-y^{2}}\right.\right. \\
& \left.\left.-x^{2}\left(4 y^{2}-1\right)\right]+\omega_{n}^{2} \tilde{r}_{n}^{2}\left[y^{2}-4 x y \sqrt{1-x^{2}} \sqrt{1-y^{2}}-x^{2}\left(4 y^{2}-1\right)\right]\right\} / \\
& \left\{\pi\left(\alpha^{\prime} p^{+}\right)^{2}\left(r_{n}^{2} x^{2}+\tilde{r}_{n}^{2} y^{2}\right)^{2}\right\} \mathrm{d} x \wedge \mathrm{d} y .
\end{aligned}
$$

Using the residue theorem and integrating first with respect to $x$ and then with respect to $y$, we get

$$
\int_{-1}^{1} \int_{-1}^{1} e(T M) \mathrm{d} x \mathrm{~d} y=\frac{\left(\omega_{n}^{2}-n^{2}\right)}{n \omega_{n}} \frac{\tilde{r}_{n}}{r_{n}}
$$

while in the inverse integration order the result is

$$
\int_{-1}^{1} \int_{-1}^{1} e(T M) \mathrm{d} y \mathrm{~d} x=\frac{\left(\omega_{n}^{2}-n^{2}\right)}{n \omega_{n}} \frac{r_{n}}{\tilde{r}_{n}}
$$

As we see, the outcome of the integral depends on the order of integration because there are points in the domain of integration where the Euler form has singularities. Analyzing the region of integration and its boundary, we find that the right

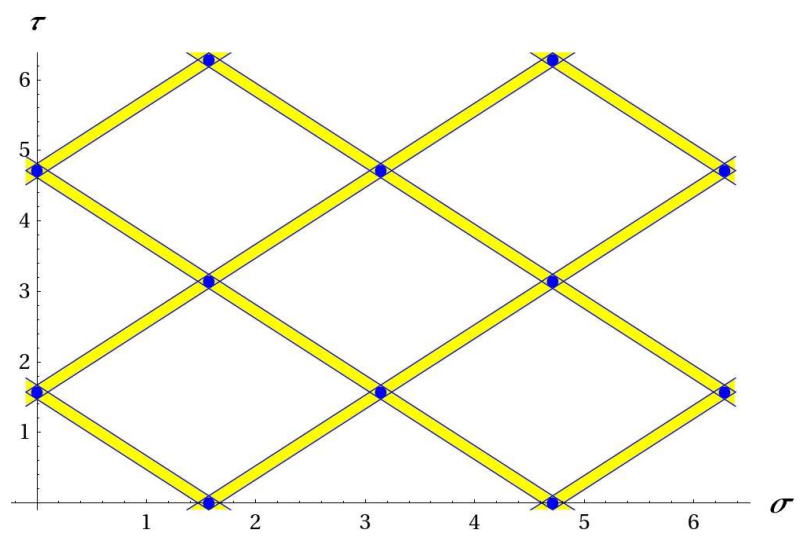

Figure 1. Plot of the region of integration in $(\sigma, \tau)$ space. The dots correspond to the singularities of Euler form (19) and the shadowed region is the area not considered in the numerical integration. 
expression for the Euler invariant should be

$$
\xi_{T M}=\int_{M} e(T M) \mathrm{d} V=\frac{1}{2} \frac{\left(\omega_{n}^{2}-n^{2}\right)}{n \omega_{n}}\left(\frac{\tilde{r}_{n}}{r_{n}}+\frac{r_{n}}{\tilde{r}_{n}}\right) .
$$

As a confirmation that (20) is correct, we integrated (19) numerically over the domain of integration for $n=1$, excluding bands of width $\epsilon$ where the singularities are located (Fig. 1). In Fig. 2, we plot $\xi_{T M}$ as a function of $r_{1}$ and $\tilde{r}_{1}$ computed by (a) using (20) and (b) the numerical method for $\epsilon=0.01$. We can see that the behavior is very similar in these two plots except for a large additive constant. We confirmed by exploration, that as $\epsilon \rightarrow 0$, the profiles of the two plots look more alike while the additive constant becomes larger. From this, we conclude that (20) is the right expression, once the divergence of the integral has been removed using the appropriate singularity theorems.
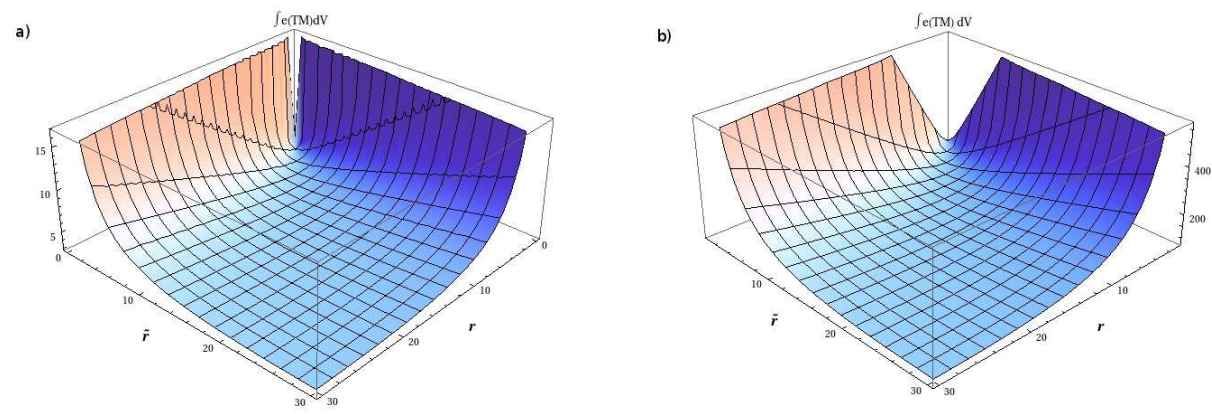

Figure 2. Plot of the Euler characteristic $\xi_{T M}(r, \tilde{r})$ computed using a) expression (20) and b) the numerical method described in the text (taking $\omega_{1}=2, p^{+}=1$ and $\left.\alpha^{\prime}=1\right)$.

Now we are in a position to apply the Gauss-Bonnet theorem to (20) to obtain the topological spectrum which is expressed as

$$
\frac{r}{\tilde{r}}+\frac{\tilde{r}}{r}=\frac{2 n \sqrt{n^{2}+\left(\mu \alpha^{\prime} p^{+}\right)^{2}}}{\left(\mu \alpha^{\prime} p^{+}\right)^{2}} k .
$$

We see that for a given mass of the bosonic field, the ratio of the amplitudes $r / \tilde{r}$ is not arbitrary but it must fulfill a discrete relation which depends on the integer $k$.

\section{Physical Consequences and Discussion}

The topological spectrum for the particular configuration under consideration for the massive scalar field describes a discrete relationship between the parameters 
that determines the field. In this work we have only considered the particular case when the frequencies $\omega_{n}$ are the same for both fields $X^{I=1,2}$, but it is not difficult to see that these relationships depend in general on the number of modes considered for the scalar field as is shown in [2]. Therefore, it is interesting to find out what characteristics of the field result are affected by this discreteness. To this end, let us compute the Hamiltonian of the system $H=\int \mathcal{H} \mathrm{d} \sigma=\int_{0}^{2 \pi \alpha^{\prime} p^{+}}\left(\sum_{A} \dot{X}^{A} \Pi_{A}-\right.$ $\mathcal{L}) \mathrm{d} \sigma$. Choosing $\tau$ as the time parameter, from equation (10) we obtain

$$
\mathcal{H}=\left(1 / 4 \pi \alpha^{\prime}\right) \sum_{I=1}^{8}\left(\partial_{\tau} X^{I} \partial_{\tau} X^{I}+\partial_{\sigma} X^{I} \partial_{\sigma} X^{I}+\mu X^{I} X^{I}\right)
$$

and thus, for the case of the particular configuration of the fields (18), we obtain

$$
H=\frac{\omega_{n}}{\alpha^{\prime} p^{+}}\left(r_{n}^{2}+\tilde{r}_{n}^{2}\right)
$$

A comparison with the topological spectrum (21) yields

$$
H=k \frac{n\left[n^{2}+\left(\mu \alpha^{\prime} p^{+}\right)^{2}\right]}{\left(\mu \alpha^{\prime} p^{+}\right)^{2}} r_{n} \tilde{r}_{n} \equiv k C_{n n} r_{n} \tilde{r}_{n}
$$

where $C_{n n}$ is a constant. This result shows that the Hamiltonian describing the free massive bosonic field has a discrete behavior proportional to $k \in \mathbb{Z}$. Also, we notice that it is proportional to the term $r_{n} \tilde{r}_{n}$ involving the amplitudes. This implies that the discrete behaviour of the Hamiltonian must be independent of the choice of the fields that are taken. This is satisfied if this product is also a constant. Surprisingly, we found that the right and left modes are not independent from each other and this implication does not have an analogue in other formalisms. As was mentioned above, this result is valid only when $\omega_{n}$ is the same for both fields, for a more general case we must refer to [2] where it is shown that the behaviour of the Hamiltonian dictated by the topological spectrum is different.

Another important feature of this physical system in the context of topological quantization is the fact that the Euler form has singularities inside the region of integration and this is responsible for the fact that the Euler invariant does not commute under the interchange of the integration variables. We can see now the integrals

$$
\int() \mathrm{d} x \equiv F_{x}, \quad \int() \mathrm{d} y \equiv G_{y}
$$

as operators that obey the relation

$$
F_{x} G_{y}-G_{y} F_{x} \neq 0
$$


This is a peculiar feature, because $F_{x}$ and $G_{y}$ are functions of $\alpha$ and $\tilde{\alpha}$ so, in a certain way, we obtain from the topological analysis of the system the non commuting behavior that in standard quantum field theory is only given through the imposition of this relation to the fields and its conjugate moment.

\section{Acknowledgements}

This work is supported by DGAPA-UNAM \# IN106110. F. Nettel acknowledges the support from DGAPA-UNAM (postdoctoral fellowship). G. Arciniega thanks to Sociedad Mexicana de Física and Departamento de Física, UNAM for the financial support for attending the XIII-th International Conference on Geometry, Integrability and Quantization held in Varna, Bulgaria in June 3-8, 2011.

\section{References}

[1] Arciniega G., Nettel F., Patino L. and Quevedo H., Topological Discretization of Bosonic Strings on Flat and Curved Backgrounds, In preparation.

[2] Arciniega G., Nettel F., Patino L. and Quevedo H., Topological Quantization of Free Massive Bosonic Fields, In preparation.

[3] Becker K., Becker M. and Schwartz J., String Theory and M-theory: A Modern Introduction, Cambridge University Press, Cambridge 2007.

[4] Blau M., Figueroa-O'Farril J, Hull C. and Papadopoulos G., A New Maximally Supersymmetric Background of IIB Superstring Theory, JHEP 1 (2002) 47-62.

[5] Courant R. and Fritz J., Introduction to Calculus and Analysis, John Wiley \& Sons, New York 1974.

[6] Dirac P., Quantised Singularities in the Electromagnetic Field, Proc. Roy. Soc. Lond. A 133 (1931) 60-72.

[7] Nettel F. and Quevedo H., Topological Spectrum of Classical Configurations, AIP Conf. Proc. 956 (2007) 9-14.

[8] Nettel F., Quevedo H. and Rodíguez M., Topological Spectrum of Mechanical System, Rep. Math. Phys. 64 (2009) 355-365.

[9] Nettel F. and Quevedo H., Topological Spectrum of the Harmonic Oscillator, Int. J. of Pure and Appl. Math. 70 (2011) 117-123.

[10] Nepomechie R., Magnetic Monopoles From Antisymmetric Tensor Gauge Fields, Phys. Rev. D 31 (1985) 1921-1924. 
[11] Patino L. and Quevedo H., Bosonic and Fermionic Behavior in Gravitational Configurations, Mod. Phys. Lett. A 18 (2003) 1331-1337.

[12] Patino L. and Quevedo H., Topological Quantization of Gravitational Fields, J. Math. Phys. 46 (2005) 022502.

[13] Penrose R., Any Spacetime Has a Plane Wave as a Limit, In: Diff. Geom. and Rel. 1976, pp 271-275.

[14] Peskin M. and Schroeder D., An Introduction to Quantum Field Theory, In: Frontiers in Physics, Addison-Wesley, New York 1995.

[15] Russo R. and Tanzini A., The Duality Between IIB String Theory on pp-wave and $N=4$ SYM: A Status Report, Class. Quant. Grav. 21 (2004) S1265.

[16] Sadri D. and Sheikh-Jabbari M., The Plane-Wave / Super Yang-Mills Duality, Rev. Mod. Phys. 76 (2004) 853-907.

[17] Teitelboim C, Monopoles of Higher Rank, Phys. Lett. B 167 (1986) 69-72.

Gustavo Arciniega

Francisco Nettel

Leonardo Patino

Departamento de Física

Facultad de Ciencias

Universidad Nacional Autónoma

de México C.P. 04510, México, D.F.

E-mail addresses:

gustavo.arciniega@gmail.com

fnettel@ciencias. unam.mx

leopj@ciencias.unam.mx
Hernando Quevedo

Instituto de Ciencias Nucleares

Universidad Nacional Autónoma

de México

C.P. 04510, México, D.F.

E-mail address:

quevedo@nucleares.unam.mx 